\title{
THE HADAMARD PRODUCT OF TWO BROWNIAN MATRICES: ANALYTIC INVERSE AND DETERMINANT
}

\author{
F. N. VALVI ${ }^{1}$
}

(Received 10 March 1993; revised 9 July 1993)

\begin{abstract}
The explicit inverse and determinant of a class of matrices is given. The class is the Hadamard product of two already known classes. Its elements are defined by $3 n-1$ parameters, analytical expressions of which compose the Hessenberg form inverse. These expressions enable a recursive formula to be obtained, which gives the inverse in $O\left(n^{2}\right)$ multiplications/divisions and $O(n)$ additions/subtractions.
\end{abstract}

\section{Introduction}

Linear models are frequently used in the theory of digital signal processing. A common characteristic of such models is that they lead to linear systems of general form $B x=c$, where $B$ denotes a generalized resultant matrix obtained from certain Brownian matrices. This is due to the fact that discrete-time Brownian motion is a proper model for discrete-time signals, of which, in turn, the covariance matrix is Brownian (see, for example, [3], [9], and references therein). Furthermore, if there are two discrete-time-signal devices, represented by their covariance Brownian matrices, $B_{1}$ and $B_{2}$, respectively, then their coupling results in a generalized resultant matrix $B=B_{1}$.op. $B_{2}$, where the operator .op. may be (i) matrix multiplication or (ii) Hadamard product. If $B_{1}^{-1}$ and $B_{2}^{-1}$ can be computed by "fast" algorithms, then the same could also be true for $B^{-1}$ in the case (i). In the present paper a fast algorithm is developed for computing $B^{-1}$ in the case (ii), when $B_{1}$ and $B_{2}$ are given Brownian matrices, $B_{1}=N$ and $B_{2}=A$.

In particular, in [10] the tridiagonal inverse of a symmetric class of matrices $N=\left[a_{i j}\right]$ with elements $a_{i j}=k_{i}, i \geq j$, has been obtained. In [11] a more general

\footnotetext{
'Department of Mathematics, University of Patras, Patras, Greece.

(C) Australian Mathematical Society, 1995, Serial-fee code 0334-2700/95
} 
type of the matrix $N$ has been presented; namely, the matrix $A=\left[a_{i j}\right]$, where

$$
a_{i j}= \begin{cases}a_{j} & i \geq j, \\ b_{i} & i<j,\end{cases}
$$

the inverse of which is of upper Hessenberg form. In this paper we present the explicit inverse and determinant of a class represented by the Hadamard product of these two classes, that is, $B=N \circ A$, where $B$ is the class under consideration and o denotes the Hadamard product.

If we consider the $n \times n$ matrix $P=\left[p_{i j}\right]$, with elements

$$
p_{i j}= \begin{cases}1 & i+j=n+1, \\ 0 & \text { otherwise, }\end{cases}
$$

then the matrix $P N P$ is a pure Brownian matrix, while $A$ is a Brownian matrix, in agreement with the definitions of [9] and [3], respectively. In [1] the so-called "diagonal innovation matrices" (DIM) are treated, special cases of which are both the matrices $N$ and $A$.

The Hadamard product of these matrices, besides its interest from both the mathematical and the digital-signal-processing viewpoint, does also constitute a significant general case of already known classes of test matrices. In particular, by assigning proper values to the $3 n-1$ parameters, a variety of matrices for testing computational algorithms can be constructed. It is worth noting that, by restricting the $a$ 's, $b$ 's, and $k$ 's, well-known classes of test matrices become subcases of the present category; namely, the classes appeared in [2], [5] - [8], [10], [11], as well as in [4], pages 42 and 48.

\section{The matrix and its inverse}

Let us consider a matrix $B=\left[b_{i j}\right]$ with elements

$$
b_{i j}= \begin{cases}k_{i} a_{j} & i \geq j \\ k_{j} b_{i} & i<j .\end{cases}
$$

Its inverse $B^{-1}=\left[\sigma_{i j}\right]$ is found to be an upper Hessenberg matrix with elements given analytically by the formulae

$$
\sigma_{i j}= \begin{cases}\left(k_{i-1} a_{i+1}-k_{i+1} b_{i-1}\right) /\left(c_{i-1} c_{i}\right) & i=j=2, \ldots, n-1, \\ a_{2} /\left(c_{0} c_{1}\right) & i=j=1, \\ k_{n-1} /\left(k_{n} c_{n-1}\right) & i=j=n, \\ (-1)^{i+j} d_{j} g_{i-1} \prod_{v=i+1}^{j-1} k_{v} f_{v} / \prod_{v=i-1}^{j} c_{v} & i<j, \\ -1 / c_{j} & i=j+1, \\ 0 & i>j+1,\end{cases}
$$


where

$$
\begin{array}{ll}
c_{i}=k_{i} a_{i+1}-k_{i+1} b_{i} & i=1,2, \ldots, n-1, c_{0}=a_{1}, c_{n}=1, \\
d_{i}=k_{i} a_{i+1}-k_{i+1} a_{i} & i=2,3, \ldots, n-1, d_{n}=1, \\
f_{i}=b_{i}-a_{i} & i=2,3, \ldots, n-1, \\
g_{i}=k_{i} b_{i+1}-k_{i+1} b_{i} & i=1,2, \ldots, n-2, g_{0}=b_{1}, \quad \text { and } \\
\prod_{\nu=i+1}^{j-1} k_{\nu} f_{\nu}=1 & \text { whenever } j=i+1 .
\end{array}
$$

Evidently, the above formulae are valid if

$$
k_{n} \neq 0 \quad \text { and } \quad c_{i} \neq 0 \quad i=0,1, \ldots, n-1 .
$$

\section{The proof}

To verify (2), we adopt a similar method to that in [11]. By applying a number of row operations to the matrix (1), we transform it to the identity matrix. Then the product of the elementary matrices gives the inverse matrix (2). By adopting the conventions (3) and $k_{0}=1$, the row operations carried out are the following:

1. row $i-k_{i} / k_{i-1}$ row $(i-1), \quad i=n, n-1, \ldots, 2$

2. row $i-k_{i} g_{i-1} /\left(k_{i-1} g_{i}\right)$ row $(i+1), \quad i=1,2, \ldots, n-2$

3. row $(n-1)-k_{n-1} g_{n-2} /\left(k_{n-2} c_{n-1}\right)$ row $n$ and row $i-k_{i} k_{i} g_{i-1} f_{i+1} /\left(k_{i-1} g_{i} c_{i}\right)$ row $(i+1), \quad i=n-2, n-3, \ldots, 1$.

4. $1 /\left(k_{1} a_{1}\right)$ row 1 and $k_{i-1} /\left(k_{i} c_{i-1}\right)$ row $i, \quad i=2,3, \ldots, n$.

The above operations transform the identity matrix to the following forms, respectively:

1. The bidiagonal matrix consisting of the main diagonal $(1,1, \ldots, 1)$ and the lower first diagonal $\left(-k_{2} / k_{1},-k_{3} / k_{2}, \ldots,-k_{n} / k_{n-1}\right)$.

2. The tridiagonal matrix with main diagonal

$\left(k_{1} b_{2} / g_{1}, k_{2}\left(k_{1} b_{3}-k_{3} b_{1}\right) /\left(k_{1} g_{2}\right), \ldots, k_{n-2}\left(k_{n-3} b_{n-1}-k_{n-1} b_{n-3}\right) /\left(k_{n-3} g_{n-2}\right), 1,1\right)$, upper first diagonal

$$
\left(-k_{1} b_{1} / g_{1},-k_{2} g_{1} /\left(k_{1} g_{2}\right), \ldots,-k_{n-2} g_{n-3} /\left(k_{n-3} g_{n-2}\right), 0\right),
$$

and lower first diagonal $\left(-k_{2} / k_{1},-k_{3} / k_{2}, \ldots,-k_{n} / k_{n-1}\right)$.

3. The upper Hessenberg matrix of the type (case $n=5$ ) 


$\left[\begin{array}{ccccc}\frac{k_{1} a_{2}}{c_{1}} & -\frac{k_{1} b_{1} d_{2}}{c_{1} c_{2}} & \frac{k_{1} b_{1} d_{3} k_{2} f_{2}}{c_{1} c_{2} c_{3}} & -\frac{k_{1} b_{1} d_{4} k_{2} f_{2} k_{3} f_{3}}{c_{1} c_{2} c_{3} c_{4}} & \frac{k_{1} b_{1} k_{2} f_{2} k_{3} f_{3} k_{4} f_{4}}{c_{1} c_{2} c_{3} c_{4}} \\ -\frac{k_{2}}{k_{1}} & \frac{k_{2}\left(k_{1} a_{3}-k_{3} b_{1}\right)}{k_{1} c_{2}} & -\frac{k_{2} d_{3} g_{1}}{k_{1} c_{2} c_{3}} & \frac{k_{2} d_{4} g_{1} k_{3} f_{3}}{k_{1} c_{2} c_{3} c_{4}} & -\frac{k_{2} g_{1} k_{3} f_{3} k_{4} f_{4}}{k_{1} c_{2} c_{3} c_{4}} \\ 0 & -\frac{k_{3}}{k_{2}} & \frac{k_{3}\left(k_{2} a_{4}-k_{4} b_{2}\right)}{k_{2} c_{3}} & -\frac{k_{3} d_{4} g_{2}}{k_{2} c_{3} c_{4}} & \frac{k_{3} g_{2} k_{4} f_{4}}{k_{2} c_{3} c_{4}} \\ 0 & 0 & -\frac{k_{4}}{k_{3}} & \frac{k_{4}\left(k_{3} a_{5}-k_{5} b_{3}\right)}{k_{3} c_{4}} & -\frac{k_{4} g_{3}}{k_{3} c_{4}} \\ 0 & 0 & 0 & -\frac{k_{5}}{k_{4}} & 1\end{array}\right]$.

4. The upper Hessenberg matrix, the elements of which are given by (2).

\section{The determinant}

The determinant of $B$ is derived easily by carrying out the row operation, row $i-$ $k_{i} / k_{i-1}$ row $(i-1), i=n, n-1, \ldots, 2$, which provides the upper triangular matrix

$$
\left[\begin{array}{ccccc}
k_{1} a_{1} & k_{2} b_{1} & k_{3} b_{1} & \ldots & k_{n} b_{1} \\
0 & k_{2} c_{1} / k_{1} & k_{3} g_{1} / k_{1} & \ldots & k_{n} g_{1} / k_{1} \\
0 & 0 & k_{3} c_{2} / k_{2} & \ldots & k_{n} g_{2} / k_{2} \\
\ldots & \ldots & \ldots & \ddots & \ldots \\
0 & 0 & 0 & \ldots & k_{n} c_{n-1} / k_{n-1}
\end{array}\right],
$$

using the abbreviations (3). Hence

$$
\operatorname{det}(B)=a_{1} k_{n}\left(k_{1} a_{2}-k_{2} b_{1}\right)\left(k_{2} a_{3}-k_{3} b_{2}\right) \ldots\left(k_{n-1} a_{n}-k_{n} b_{n-1}\right),
$$

which yields the criteria for the singularity of the matrix $B$; that is,

$$
k_{n}=0 \quad \text { or } \quad c_{i}=0 \quad \text { for some } i \in\{0,1, \ldots, n-1\} .
$$

\section{Numerical complexity}

The formulae in (2), which give the elements of the inverse matrix above the main diagonal, enable us to get a recursion relation in order to facilitate the evaluation of $B^{-1}$. This relation provides the recursive algorithm

$$
\begin{array}{rlrl}
\sigma_{i, i+1} & =-\frac{d_{i+1} g_{i-1}}{c_{i-1} c_{i} c_{i+1}} & i & =1,2, \ldots, n-1, \\
\sigma_{i, i+s+1} & =-\frac{d_{i+s+1} k_{i+s} f_{i+s}}{d_{i+s} c_{i+s+1}} \sigma_{i, i+s} & i & =1,2, \ldots, n-2, s=1,2, \ldots, n-i-1,
\end{array}
$$


or, alternatively,

$$
\begin{array}{rlrl}
\sigma_{j-1, j} & =-\frac{d_{j} g_{j-2}}{c_{j-2} c_{j-1} c_{j}} & j=2,3, \ldots, n, \\
\sigma_{j-s-1, j} & =-\frac{g_{j-s-2} k_{j-s} f_{j-s}}{g_{j-s-1} c_{j-s-2}} \sigma_{j-s, j} & j=3,4, \ldots, n, s=1,2, \ldots, j-2 .
\end{array}
$$

The above formulae estimate all the elements $\sigma_{i j}$, for $i<j$, and reduce the number of multiplications/divisions for the evaluation of the inverse matrix to $n^{2} / 2+29 n / 2-$ 25 in all, since the coefficient of $\sigma_{i, i+s}\left(\sigma_{j-s, j}\right)$ depends only on the second (first) subscript. Finally, the number of additions/subtractions is $5 n-9$.

\section{References}

[1] G. Carayannis, N. Kalouptsidis and D. G. Manolakis, "Fast recursive algorithms for a class of linear equations", IEEE Trans. Acoust. Speech Signal Process. 30 (1982) 227-239.

[2] W. L. Frank, "Computing eigenvalues of complex matrices by determinant evaluation and by methods of Danilewski and Wielandt", SIAM J. 6 (1958) 378-392.

[3] M. J. C. Gover and S. Barnett, "Brownian matrices: properties and extensions", Internat. J. Systems Sci. 17 (1986) 381-386.

[4] R. T. Gregory and D. L. Karney, A collection of matrices for testing computational algorithms (Wiley-Interscience, New York, 1969).

[5] P. Konavic, "Inversion of a covariance matrix", J. Comput. Phys. 5 (1970) 355-357.

[6] M. H. Lietzke, R. W. Stoughton and M. P. Lietzke, "A comparison of several methods for inverting large symmetric positive definite matrices", Math. Comp. 18 (1964) 449-456.

[7] H. W. Milnes, "A note concerning the properties of a certain class of test matrices", Math. Comp. 22 (1968) 827-832.

[8] M. Newman and J. Todd, "The evaluation of matrix inversion programs", SIAM J. 6 (1958) 466-476.

[9] B. Picinbono, "Fast algorithms for Brownian matrices", IEEE Trans. Acoust. Speech Signal Process. 31 (1983) 512-514.

[10] F. N. Valvi, "Explicit presentation of some types of matrices", J. Inst. Math. Appl. 19 (1977) 107-117.

[11] F. N. Valvi and V. S. Geronyannis, "Analytic inverses and determinants for a class of matrices", IMA J. Numer. Anal. 7 (1987) 123-128. 